\title{
Streptococcus agalactiae: colonização de gestantes de alto risco em um hospital regional da Amazônia brasileira e perfil de sensibilidade aos antimicrobianos
}

\section{Streptococcus agalactiae: colonization of high-risk pregnant women in a regional hospital in the Brazilian Amazon and antimicrobial sensitivity profile}

Edlainny Araujo Ribeiro' (D), Georgia Miranda Tomich' ${ }^{(D)}$, Brena de Almeida Costa' (iD, Rodrigo Alves de Oliveira' (iD, Lorrany Karen Batista de Jesus' (iD)

' Faculdade de Ensino Superior da Amazônia Reunida, Redenção, Pará, Brasil

\begin{abstract}
RESUMO
INTRODUÇÃO: Estreptococo do grupo B (EGB) ou Streptococcus agalactiae em indivíduos imunossuprimidos, como os neonatos, pode resultar em uma série de complicações e doenças, podendo levar até à morte. OBJETIVOS: Caracterizar o perfil clínico-epidemiológico de gestantes colonizadas por S. agalactiae e determinar o perfil de sensibilidade antimicrobiana dos isolados em um hospital na Amazônia. MATERIAIS E MÉTODOS: As coletas dos espécimes clínicos foram realizadas no período de 15 de março a 15 de abril de 2019 considerando as diretrizes do Centers for Disease Control and Prevention. A identificação fenotípica foi realizada de acordo com as recomendações da Agência Nacional de Vigilância Sanitária, e para o teste de sensibilidade antimicrobiana, foram seguidas as especificações do Clinical and Laboratory Standards Institute. RESULTADOS: A colonização por EGB foi detectada em 34,0\% das gestantes; as doenças crônicas mais frequentes foram hipertensão $(26,0 \%)$ e diabetes $(10,0 \%)$. Os antimicrobianos linezolida, vancomicina e meropeném foram os mais eficazes contra as bactérias. Verificou-se alta taxa de resistência para ciprofloxacina (82,4\%) e cloranfenicol (70,6\%). Das cepas analisadas, 88,2\% eram multirresistentes. CONCLUSÃO: A presença de EGB entre as gestantes de alto risco e a detecção de cepas multirresistentes, inclusive com resistência a penicilinas e cefalosporinas, traz à tona a importância da triagem para a detecção dessa bactéria durante a gestação e o início da antibioticoprofilaxia, ressaltando a necessidade de adequar a prática de acompanhamento pré-natal local às recomendações vigentes.
\end{abstract}

Palavras-chave: Streptococcus agalactiae; Gestantes; Resistência Microbiana a Medicamentos.

\begin{abstract}
INTRODUCTION: Group B streptococcus (GBS) or Streptococcus agalactiae in immunosuppressed individuals, such as neonates, can result in a series of complications and diseases, which can even lead to death. OBJECTIVES: To characterize the clinical-epidemiological profile of pregnant women colonized by S. agalactiae and determine the isolates' sensitivity profile in a hospital in the Amazon. MATERIALS AND METHODS: Clinical specimens were collected from March 15 to April 15, 2019, following the Centers for Disease Control and Prevention guidelines. The phenotypic identification was performed according to the Brazilian Health Regulatory Agency (Anvisa), and for the antimicrobial sensitivity testing, the Clinical and Laboratory Standards Institute specifications were followed. RESULTS: Colonization by GBS was found in $34.0 \%$ of the pregnant women; the most frequent chronic diseases were hypertension (26.0\%) and diabetes (10.0\%). The antimicrobials linezolid, vancomycin, and meropenem were the most effective against the bacteria. There was a high resistance rate for ciprofloxacin (82.4\%) and chloramphenicol (70.6\%); 88.2\% of the strains analyzed were multidrug-resistant. CONCLUSION: The presence of GBS among high-risk pregnant women and the detection of multidrug-resistant strains, including those with resistance to penicillins and cephalosporins, bring up the importance of screening for the detection of this bacteria during pregnancy and the beginning of antibiotic prophylaxis, emphasizing the need to adapt the practice of local prenatal care to the current recommendations.
\end{abstract}

Keywords: Streptococcus agalactiae; Pregnant Women; Microbial Drug Resistance.

\footnotetext{
Correspondência / Correspondence:

Edlainny Araujo Ribeiro

Faculdade de Ensino Superior da Amazônia Reunida, Curso Biomedicina

Av. Brasil, 1435. Bairro: Alto Paraná. CEP: 68550-0325 - Redenção, Pará, Brasil - Tel.: +55 (94) 99178-0799

E-mail: dyy_araujo77@hotmail.com
} 


\section{INTRODUÇÃO}

Streptococcus agalactiae ou estreptococo do grupo $B$ de Lancefield (EGB) apresenta morfologia de cocos em cadeia e reação tintorial gram-positiva. Pode colonizar os tratos gastrointestinal e geniturinário de mulheres, de forma assintomática, e causar infecções graves em recém-nascidos ${ }^{1,2}$. Quando transferido no momento do parto para o neonato, pode causar sepses e meningites, aumentando os riscos de mortalidade ${ }^{3}$. $\bigcirc$ Centers for Disease Control and Prevention (CDC) trata a infecção/colonização por essa bactéria como uma das principais causas infecciosas de morbidade e mortalidade neonatal nos Estados Unidos da América ${ }^{4}$. Um estudo realizado em Moçambique com um total de 183 natimortos detectou EGB em 2,1\% deles por meio de análise do sangue umbilical $^{5}$.

As complicações que se manifestam durante a gravidez, parto e puerpério podem ser prevenidas através da assistência pré-natal, sendo possível identificar situações de risco para mãe e feto ${ }^{6}$. No entanto, uma pesquisa realizada com 100 gestantes, com o intuito de detectar a colonização por EGB, demonstrou positividade de $14 \%{ }^{7}$. Outro estudo realizado no estado do Rio de Janeiro revelou que dentre as 3.647 gestantes participantes entre a $35^{a}$ e a $37^{a}$ semana gestacional, $26,2 \%$ estavam colonizadas por essa bactéria ${ }^{8}$.

Dessa forma, com o objetivo de reduzir os danos perinatais associados, Toyofuku et al. ${ }^{9}$ demonstraram que a profilaxia intraparto em gestantes colonizadas reduziu em 32,5\% a morte neonatal em uma região com altas taxas de prevalência dessa bactéria. Assim, - $\mathrm{CDC}^{4}$ recomenda a triagem para a colonização de EGB em mulheres entre a $35^{a}$ e a $37^{a}$ semana gestacional e o início da antibioticoprofilaxia assim que for identificada a presença de EGB. Já o Ministério da Saúde recomenda a investigação dessas bactérias apenas em gestantes de alto risco que apresentarem sinais e sintomas, como sangramentos. Entretanto, a gestante pode estar colonizada e não apresentar sintomas, podendo transmitir esse microrganismo para o feto, o qual ficará suscetível à infecção que pode levar à ocorrência de sepse $\mathrm{e}^{10}$.

É preciso salientar que a gestação de alto risco é caracterizada por maior suscetibilidade a prejuízos à saúde da mãe e do feto, como complicações no trabalho de parto (parto prematuro), doenças clínicas maternas e alterações fetais que podem desencadear complicações nesse período ${ }^{11}$. Adicionalmente, fatores sociodemográficos, como baixos níveis de renda, escolaridade, elevadas taxas de gestações e assistência obstétrica falha favorecem a persistência dos indicadores de mortalidade entre as gestantes ${ }^{12,13,14}$.

Além disso, bactérias do gênero Streptococcus podem apresentar resistência aos antimicrobianos de escolha profilática, devido a alterações químicas nos sítios de ligação dos antimicrobianos na parede celular e transferência de genes codificadores de resistência ${ }^{15}$. Podem ainda formar comunidades que propiciam sua sobrevivência em ambientes hostis por longos períodos, chamados biofilmes ${ }^{15}$. Desse modo, essa bactéria é uma importante causa de infecção em mulheres grávidas e seus recém-nascidos; apesar disso, tem sido pouco estudada na América Latina ${ }^{16}$.

O conhecimento sobre a colonização e o perfil de sensibilidade de EGB isolados em gestantes é de grande valia, considerando a possibilidade de transmissão vertical, a sua patogenicidade, os riscos inerentes à saúde da mãe e do feto e a possibilidade de resistência aos antimicrobianos. Além desses fatores, cabe ressaltar que, durante o período gestacional, a administração de antimicrobianos é complicada, principalmente tratando-se de gestantes de alto risco; e, no estado do Pará, Brasil, há escassez de dados epidemiológicos sobre esse assunto.

Diante do exposto, esta pesquisa teve como objetivos caracterizar o perfil clínico-epidemiológico de gestantes colonizadas por S. agalactiae e determinar o perfil de sensibilidade desses isolados em um hospital que presta serviços de média e alta complexidade na Amazônia

\section{MATERIAIS E MÉTODOS}

\section{ASPECTOS ÉTICOS}

A pesquisa foi aprovada pelo Comitê de Ética em Pesquisa do Sul do Pará, pertencente à Faculdade de Ensino Superior da Amazônia Reunida, no dia 29 de outubro de 2018, sob o número CAAE: 99503018.2.0000.8104; e, atendendo à Resolução n 466/12 do Conselho Nacional de Saúde, as coletas só foram iniciadas no mês de março de 2019, após aprovação pelo Comitê.

\section{TIPO E LOCAL DO ESTUDO}

estudo foi do tipo descritivo, prospectivo e transversal, com abordagem quantitativa, realizado em um hospital público regional, referência em gestação de alto risco, que atende em média cerca de 100 gestantes por mês e presta serviços de média e alta complexidade a pacientes de 15 municípios do sudeste do estado do Pará, os quais pertencem ao $12^{\circ}$ Centro Regional de Saúde, que engloba uma população de 541.347 habitantes. $\bigcirc$ hospital está situado na cidade de Redenção, à distância de 1.018 km da capital Belém ${ }^{17,18}$.

\section{PERÍODO E POPULAÇÃO DO ESTUDO}

As coletas foram realizadas no período de 15 de março a 15 de abril de 2019, totalizando 32 dias consecutivos. Para a seleção da população deste estudo, foram considerados os seguintes critérios de inclusão: mulher em acompanhamento pré-natal no hospital que apresentava gestação de alto risco. Apesar do CDC $^{4}$ recomendar a realização do exame a partir da $35^{a}$ semana gestacional, para fins desta pesquisa foram incluídas gestantes a partir da $22^{a}$. Atendendo à resolução do Conselho Nacional de Saúde $n^{\circ} 466 / 12^{19}$, todas as gestantes que concordaram em participar do estudo assinaram o Termo de Consentimento Livre e Esclarecido (TCLE). Foram 
excluídas as gestantes que não assinaram o TCLE, as que não possuíam capacidade civil e dependiam da assinatura do seu tutor ou curador, bem como as que faziam uso de antibiótico e/ou que utilizaram nos últimos 10 dias.

Assim, 50 gestantes participaram do estudo. Dois swabs estéreis alginatados foram coletados de cada uma delas, um retal e outro vaginal, os quais foram imediatamente encaminhados para análise no laboratório do próprio hospital, seguindo recomendações posteriormente descritas.

Os dados sociodemográficos e clínico-obstétricos foram analisados considerando as variáveis: raça, escolaridade, moradia (rural/urbana), município de origem, faixa etária, doenças crônicas (de base), infecções sexualmente transmissíveis (IST), número de gestações e idade gestacional no momento da coleta dos swabs.

\section{PROCEDIMENTOS PARA COLETA, DETECÇÃO FENOTÍPICA E PERFIL DE SENSIBILIDADE}

As coletas dos espécimes clínicos foram realizadas de acordo com as recomendações do $\mathrm{CDC}^{4}$. Utilizando swabs alginatados estéreis, foram obtidas amostras do introito vaginal e do reto. $\bigcirc$ material coletado foi imediatamente inoculado em caldo de enriquecimento seletivo Todd Hewitt $\left(\operatorname{Probac}^{\circledR}\right.$ ) suplementado com antibióticos, hermeticamente fechado e levado até o laboratório do próprio hospital.

No setor de microbiologia, as amostras inoculadas foram incubadas a $35-37{ }^{\circ} \mathrm{C}$, por $24 \mathrm{~h}$; posteriormente, foram repicadas em Todd Hewitt Sangue $\left(\operatorname{Probac}^{\circledR}\right)$, sendo adicionada primeiro uma fita Hemolisinabac (Probac ${ }^{\circledR}$ ) no centro da placa. Com o auxílio de uma alça bacteriológica estéril e descartável, foram realizadas estrias perpendiculares à fita, e, logo após, as placas foram incubadas novamente a 35-37 ${ }^{\circ} \mathrm{C}$, por $24 \mathrm{~h}$. Utilizando controle positivo para teste de CAMP após $24 \mathrm{~h}$, realizou-se a interpretação do teste com detecção de $\beta$-hemólise com formação de seta. Seguindo as recomendações da Agência Nacional de Vigilância Sanitária para a identificação fenotípica de S. agalactiae 20 , as cepas foram submetidas à prova da catalase e ao teste de PYR $\left(\operatorname{Probac}^{\circledR}\right)$, para determinação da atividade da enzima pyrrolidonil arilamidase, nos quais observou-se negatividade para todas as cepas incluídas nesta pesquisa.

teste de sensibilidade aos antimicrobianos foi realizado a partir de culturas recentes, com suspensões das amostras em solução salina esterilizada, com turvação correspondendo a 0,5 da escala de Mac Farland em ágar sangue, pelo método de disco-difusão conforme descrito pelo Clinical and Laboratory Standards Institute $(\mathrm{CLSI})^{21}$. Como controle, foi usada uma cepa de Streptococcus pneumoniae (ATCC $^{\circledR}$ 49619 $\left.{ }^{\mathrm{TM}}\right)$. Após $24 \mathrm{~h}$ de incubação a 35-37 ${ }^{\circ} \mathrm{C}$, - diâmetro da zona de inibição de crescimento foi registrado em milímetros e os isolados foram classificados como sensíveis, intermediários e resistentes, de acordo com os critérios do $\mathrm{CLSI}^{21}$. Para a verificação da resistência induzida à clindamicina, foi realizado o D-teste, no qual o disco de clindamicina (Polisensidisc ${ }^{\circledR}$ ) foi adicionado em um sítio diferente para melhor visualização.

Foram testados os antimicrobianos penicilina (10 U), ampicilina (10 $\mu \mathrm{g})$, cefotaxima (30 $\mu \mathrm{g})$, eritromicina $(15 \mu \mathrm{g})$, clindamicina $(2 \mu \mathrm{g})$, cloranfenicol (30 $\mu \mathrm{g})$, oxacilina (1 $\mu \mathrm{g})$, azitromicina (15 $\mu \mathrm{g})$, ciprofloxacina $(5 \mu \mathrm{g})$, tetraciclina $(30 \mu \mathrm{g})$, linezolida $(30 \mu \mathrm{g})$, vancomicina $(30 \mu \mathrm{g})$ e meropeném $(10 \mu \mathrm{g})^{22}$. A resistência a três ou mais classes dos antimicrobianos testados foi definida como resistência a múltiplos fármacos ${ }^{22}$.

\section{ANÁLISE DOS DADOS}

Após a coleta, os dados foram organizados em tabelas do Microsoft Excel (2013) e consolidados de acordo com a codificação apropriada para cada uma das variáveis estudadas. Foi realizada a análise descritiva dos dados utilizando frequência absoluta, relativa (percentual), média e desvio padrão.

\section{RESULTADOS}

Foram rastreadas amostras de secreções vaginais e retais de 50 gestantes de alto risco atendidas em um hospital regional no sudeste do estado do Pará para a presença de S. agalactiae. A colonização se fez presente em 34,0\% (17/50) das gestantes, das quais $35,3 \%(6 / 17)$ ocorreram em secreções vaginais, $23,5 \%$ (4/17) em região anal e 41,2\% (7/17) em ambas. A média de idade das participantes foi de 26,4 anos (desvio padrão $\pm 7,2$ ). A maior frequência de exames positivos foi observada em gestantes na fase termo, com escolaridade $\leq$ oito anos, cor de pele não branca, residente em zona rural e que apresentavam multigestações (Tabela 1).

Dentre os fatores que justificam o acompanhamento dessas gestantes em atendimento de média e alta complexidade, os mais frequentes observados foram hipertensão $(26,0 \% ; 13 / 50)$, diabetes $(10,0 \% ; 5 / 50)$, gestação gemelar $(10,0 \%$; 5/50), doenças renais $(8,0 \% ; 4 / 50)$ e idade materna $(8,0 \% ; 4 / 50)$. Outro risco associado foi a presença de IST, como sífilis, hepatite viral B e infecção pelo HIV, que foi observado em 6,0\% (3/50) das gestantes.

Com relação ao perfil de sensibilidade dos microrganismos isolados, $100,0 \% \quad(17 / 17)$ se mostraram sensíveis aos antimicrobianos linezolida, vancomicina e meropeném (Figura 1). Porém, altas taxas de resistência foram detectadas para ciprofloxacina (82,4\%; 14/17), seguido por cloranfenicol (70,6\%; 12/17), azitromicina (58,8\%; 10/17) e tetraciclina (58,8\%; 10/17). Das cepas analisadas, $88,2 \% \quad(15 / 17)$ eram multirresistentes, apresentando resistência à eritromicina, ampicilina, penicilina, ciprofloxacina e tetraciclina (Quadro 1). As classes de antimicrobianos com maiores taxas de resistência (in vitro) foram as quinolonas, seguidas por $\beta$-lactâmicos, anfenicóis, macrolídeos, lincosamidas e tetraciclinas. 
Tabela 1 - Perfil sociodemográfico, fatores clínico-obstétricos e frequência de gestantes colonizadas por EGB, assistidas em um hospital regional do estado do Pará, Brasil, entre março e abril de 2019

\begin{tabular}{|c|c|c|c|c|}
\hline \multirow{2}{*}{ Variáveis de exposição } & \multicolumn{2}{|c|}{ Gestantes } & \multicolumn{2}{|c|}{ Exames positivos } \\
\hline & $N=50$ & $\%$ & $N=17$ & $\%$ \\
\hline \multicolumn{5}{|l|}{ Idade $(X=26,4$; desvio padrão $\pm 7,2)$} \\
\hline$\leq 25$ & 24 & 48,0 & 8 & 33,3 \\
\hline$\geq 26$ & 26 & 52,0 & 9 & 34,6 \\
\hline \multicolumn{5}{|l|}{ Escolaridade } \\
\hline$\leq 8$ anos & 9 & 18,0 & 5 & 55,6 \\
\hline$\geq 9$ anos & 41 & 82,0 & 12 & 29,3 \\
\hline \multicolumn{5}{|l|}{ Cor de pele } \\
\hline Branca & 4 & 8,0 & 1 & 25,0 \\
\hline Não branca & 46 & 92,0 & 16 & 34,8 \\
\hline \multicolumn{5}{|l|}{ Área de residência } \\
\hline Rural & 7 & 14,0 & 4 & 57,1 \\
\hline Urbana & 43 & 86,0 & 13 & 30,2 \\
\hline \multicolumn{5}{|l|}{ No de gestações } \\
\hline Primigestas & 21 & 42,0 & 6 & 28,6 \\
\hline Multigestas & 29 & 58,0 & 11 & 37,9 \\
\hline \multicolumn{5}{|c|}{ Idade gestacional (momento da coleta do swab) } \\
\hline \multicolumn{5}{|l|}{$(X=31,9 ;$ desvio padrão $\pm 5,9)$} \\
\hline Pré-termo $<37$ semanas & 35 & 70,0 & 10 & 28,6 \\
\hline Termo 37 a 42 semanas & 15 & 30,0 & 7 & 46,7 \\
\hline Pós-termo $>42$ semanas & - & - & - & - \\
\hline \multicolumn{5}{|l|}{ Diagnóstico de IST (na gestação atual) } \\
\hline Sim & 3 & 6,0 & - & - \\
\hline Não & 47 & 94,0 & 17 & 36,2 \\
\hline
\end{tabular}

Sinal convencional utilizado: - Dado numérico igual a zero, não resultante de arredondamento.

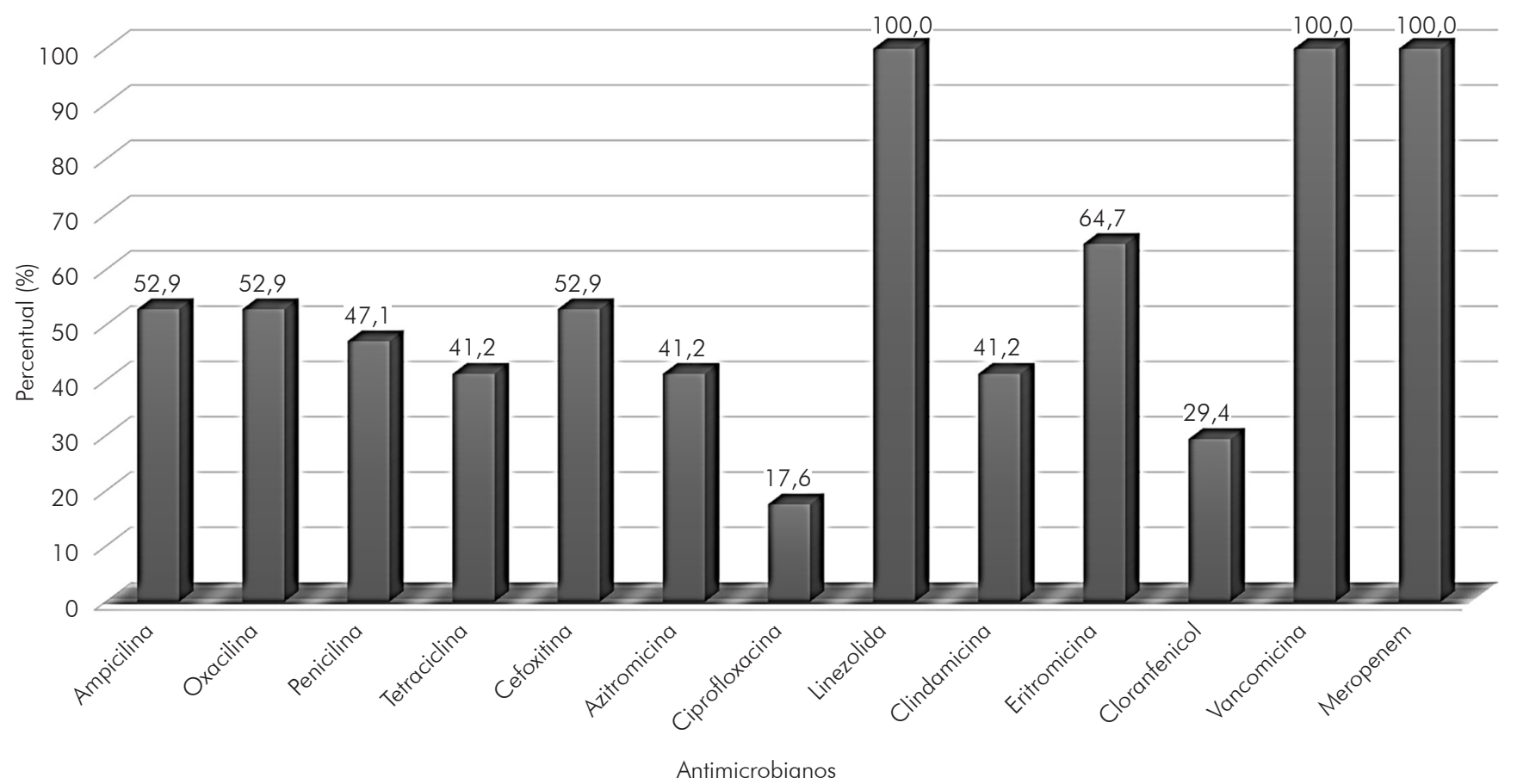

Figura 1 - Perfil de sensibilidade frente aos antimicrobianos testados em disco-difusão (in vitro) das cepas de Streptococcus agalactiae isoladas de gestantes de alto risco no sudeste do estado Pará, Brasil, entre março e abril de 2019 


\begin{tabular}{|c|c|c|c|c|c|c|c|c|c|c|c|c|c|}
\hline \multirow{2}{*}{ Amostras positivas } & \multicolumn{13}{|c|}{ Antimicrobianos } \\
\hline & AMP & OXA & PEN & TET & CFO & AZI & CIP & LNZ & $\mathrm{CLI}$ & ERI & $\mathrm{CLO}$ & VAN & MER \\
\hline $\mathrm{A} 1$ & $S$ & $S$ & $S$ & $S$ & $S$ & $R$ & $S$ & $S$ & $R$ & $S$ & $R$ & $S$ & $S$ \\
\hline A2 & S & $S$ & S & S & $S$ & $R$ & $S$ & $S$ & $R$ & $R$ & $R$ & $S$ & $S$ \\
\hline A6 & R & $R$ & $R$ & $R$ & S & S & $R$ & S & S & S & S & S & S \\
\hline A8 & $S$ & $S$ & $S$ & S & $S$ & $S$ & $R$ & $S$ & S & $S$ & $R$ & $S$ & S \\
\hline A9 & $R$ & $R$ & $R$ & $R$ & S & S & $R$ & S & S & S & S & S & S \\
\hline Al1 & S & $S$ & S & $R$ & $S$ & $R$ & $S$ & $S$ & S & $S$ & $S$ & $S$ & S \\
\hline $\mathrm{A} 12$ & $S$ & $R$ & $R$ & S & $S$ & $S$ & $R$ & S & $R$ & S & $R$ & $S$ & S \\
\hline A15 & $R$ & $R$ & $R$ & S & $R$ & S & $R$ & S & $R$ & S & $R$ & $S$ & $S$ \\
\hline Al6 & $S$ & $S$ & $S$ & $R$ & $R$ & $S$ & $R$ & $S$ & $R$ & $S$ & $R$ & $S$ & $S$ \\
\hline A21 & $S$ & $S$ & $S$ & $S$ & $R$ & $S$ & $R$ & $S$ & $R$ & $S$ & $R$ & $S$ & $S$ \\
\hline A23 & $R$ & $R$ & $R$ & $S$ & $R$ & $R$ & $R$ & $S$ & $R$ & $S$ & $R$ & $S$ & $S$ \\
\hline A24 & $S$ & $S$ & $S$ & $R$ & $R$ & $R$ & $R$ & $S$ & $R$ & $R$ & $R$ & $S$ & $S$ \\
\hline A25 & $R$ & $R$ & $R$ & $R$ & $R$ & $R$ & $R$ & $S$ & $S$ & $R$ & $S$ & $S$ & $S$ \\
\hline A28 & $R$ & $\mathrm{R}$ & $R$ & $R$ & $R$ & $R$ & $R$ & $S$ & $S$ & $S$ & $R$ & $S$ & $S$ \\
\hline A36 & $R$ & $S$ & $R$ & $R$ & $R$ & $R$ & $R$ & $S$ & $S$ & $R$ & $S$ & $S$ & $S$ \\
\hline A38 & $S$ & $S$ & $S$ & $R$ & $S$ & $R$ & $R$ & $S$ & $R$ & $R$ & $R$ & $S$ & $S$ \\
\hline A45 & $R$ & $R$ & $R$ & $R$ & $S$ & $R$ & $R$ & $S$ & $R$ & $R$ & $R$ & $S$ & $S$ \\
\hline
\end{tabular}

A: Dado demonstrativo representando as amostras positivas para Streptococcus agalactiae, nomeadas de acordo com a identificação recebida durante análise; S: Sensível; R: Resistente. AMP: Ampicilina; OXA: Oxacilina; PEN: Penicilina; TET: Tetraciclina; CFO: Cefotaxima AZI: Azitromicina; CIP: Ciprofloxacina; LNZ: Linezolida; CLI: Clindamicina; ERI: Eritromicina; CLO: Cloranfenicol; VAN: Vancomicina; MER: Meropeném.

Quadro 1 - Perfil de sensibilidade de Streptococcus agalactiae isolados de 17 gestantes de alto risco no estado do Pará, Brasil, entre março e abril de 2019

\section{DISCUSSÃO}

Neste estudo, a colonização por EGB foi detectada em gestantes de alto risco. Esse fato pode ser associado a alterações anatômicas e fisiológicas, como hipertrofia das paredes da vagina, o aumento do fluxo sanguíneo, alterações do $\mathrm{pH}$, temperatura e acidez vaginal, além de alguns hábitos de higiene íntima como, por exemplo, o uso de duchas e sabonetes íntimos ${ }^{23,24}$.

Apesar de a amostragem ser um fator limitante nesta pesquisa, estudos com o intuito de analisar os fatores sociodemográficos são capazes de inferir a existência de grupos mais suscetíveis à colonização por EGB25,26. Tal fato foi demonstrado em um estudo conduzido na Arábia Saudita com 1.328 gestantes, no qual a colonização por EGB foi de 4,2\% em gestantes com idade entre 25 e 29 anos e de $27,4 \%$ entre aquelas com mais de 40 anos $^{27}$.

Os resultados da presente pesquisa concordam com os de um estudo realizado no Rio de Janeiro, que demonstrou que hipertensão arterial e diabetes gestacional foram as patologias maternas mais observadas entre gestantes de alto risco ${ }^{8}$. A patologia que mais predispõe à colonização materna ainda não está bem elucidada; porém, pressupõe-se que a alteração do estado imunológico, como ocorre na diabetes durante a gravidez, possibilita a infecção invasiva por EGB28,29,30.

Os medicamentos utilizados para o tratamento do EGB devem ter a capacidade de inibir a síntese da parede celular, a síntese proteica e a DNA girase; todavia, alguns antimicrobianos não podem ser prescritos durante a gestação por apresentarem capacidade de romper a barreira placentária e causar prejuízos à saúde da mãe e do feto. Dessa forma, recomenda-se a utilização de penicilina, amoxacilina, ampicilina, cefalexina e nitrofurantoína durante a gestação e, em caso de alergias à penicilina, a clindamicina ou eritromicina ${ }^{4,31}$.

Entretanto, ao analisar os resultados relativos à eficácia desses antimicrobianos para os isolados desta pesquisa, foi possível observar que as penicilinas e as cefalosporinas testadas não apresentaram boa sensibilidade (in vitro). Corroborando esse dado, um estudo realizado no estado do Ceará demonstrou que dentre cepas de EGB isoladas algumas apresentavam resistência à ampicilina (44,4\%), cefalotina $(44,4 \%)$, clindamicina $(77,8 \%)$, cloranfenicol $(11,1 \%)$, eritromicina $(33,3 \%)$ e penicilina $(44,4 \%)^{32}$. Baixos índices de resistência dessa bactéria à eritromicina foram relatados em outras pesquisas, revelando índices de sensibilidade para esse medicamento de $85,7 \%$ e $92,5 \% 33,34$.

Índices de sensibilidade reduzida de EGB à penicilina foram descritos na literatura internacional ${ }^{35,36}$. Esses índices podem ser associados a diversos fatores, como o seu uso em larga escala e mutações que alteram o sítio de ligação das proteínas ligadoras de penicilinas, mutações essas que podem ser carreadas por plasmídeos e transferidas de forma horizontal entre 
espécies distintas ${ }^{35,36}$. Todavia, a descrição de resistência de EGB a esse medicamento no Brasil continua muito baixa, e isso pode estar relacionado aos baixos índices de pesquisas voltadas para essa temática e a ausência de estrutura para a realização do teste ${ }^{16,32}$. Assim como nesta pesquisa, Linhares et al. ${ }^{32}$ relataram a não realização da pesquisa do EGB para as gestantes em rotinas pré-natais.

Além disso, como demonstrado em um estudo realizado no estado do Amazonas, as penicilinas e cefalosporinas estão entre os principais medicamentos utilizados, com destaque para cefalexina $(39,7 \%)$, amoxicilina $(29,4 \%)$, seguidos por benzilpenicilina benzatina $(4,4 \%)$, ciprofloxacina $(3,7 \%)$, sulfadiazina $(3,7 \%)$, tetraciclina $(3,7 \%)$, azitromicina $(2,9 \%)$ e levofloxacina $(2,9 \%)$, o que pode influenciar o aumento de detecções de cepas resistentes a esses medicamanetos $^{37}$.

As bactérias analisadas nesta pesquisa apresentaram altos índices de resistência para a quinolona testada. Segundo uma pesquisa realizada na Argentina, esse fato está associado à ocorrência de mutações pontuais nos genes gyrA e parC ${ }^{38}$. Ressalta-se a relevância do monitoramento da sensibilidade dessa bactéria, pois, além de apresentar fatores de virulência que facilitam o desenvolvimento de processo infeccioso, pode apresentar mecanismos de resistência que dificultam a abordagem terapêutica ${ }^{38,39}$.

$\bigcirc$ uso de medicamentos de forma indiscriminada pode promover o surgimento de bactérias multirresistentes, as quais estão associadas a índices elevados de morbimortalidade ${ }^{40,41}$. Com o advento da resistência bacteriana, a identificação do patógeno e o conhecimento sobre o seu perfil de sensibilidade em infecções/colonizações durante a gestação tornaram-se importantes para uma profilaxia intraparto eficiente. Nesse sentido, o CDC recomenda uma triagem baseada em resultados microbiológicos, com detecção de colonização por coleta de swab realizada rotineiramente entre a $35^{a}$ e a $37^{a}$ semana gestacional $^{4,41}$.

De forma semelhante, o American College of Obstetricians and Gynecologists recomenda a realização do rastreio de EGB entre a $36^{a}$ e a $37^{a}$ semana de gestação, com profilaxia antibiótica intraparto apropriada ${ }^{42}$. Entretanto, neste estudo, foram consideradas as gestantes a partir da $22^{a}$ semana gestacional, levando-se em conta: o risco de parto prematuro, por se tratar de gestantes de alto risco; o fato da colonização por EGB durante a gravidez ser transitória, intermitente ou constante; e que, no hospital em estudo, a pesquisa para a presença EGB não é realizada em qualquer fase da gestação. Assim, cabe ressaltar que há evidências científicas que sugerem a associação entre a colonização por essa bactéria e a prematuridade, resultando em aumento das taxas de mortalidade ${ }^{43,44}$.

No hospital onde foi realizado o presente estudo, apesar de atender especificamente gestantes de alto risco, o exame para a detecção de EGB não é realizado como parte do acompanhamento ao pré-natal, o que pode elevar os riscos à saúde da mãe e do feto. Em contrapartida, em um hospital do estado de São Paulo, foram implementadas estratégias preventivas considerando períodos corretos para coleta, maiores índices de positividade e riscos inerentes à saúde da mãe e do feto para a triagem e detecção do EGB, possibilitando mitigar o número de infecções neonatais de $57,8 \%$ para 55,1\%, o que mostra a importância de políticas e estratégias públicas para melhoria da assistência em saúde ${ }^{44,45}$.

É importante frisar ainda que a interpretação dos resultados se deu à luz de limitações. $\bigcirc$ número de participantes foi relativamente pequeno, limitando assim a possibilidade de representatividade das detecções de resistências. Isso pode estar associado ao fato do hospital atender apenas gestantes de alto risco.

\section{CONCLUSÃO}

Foi possível evidenciar a presença de EGB entre as gestantes de alto risco atendidas em um hospital regional em uma cidade no sudeste do Pará, e detectar cepas multirresistentes, inclusive com resistência a penicilinas e cefalosporinas. As frequências de positividade para colonização por EGB e de multirresistência detectadas ressaltam a importância da triagem para detecção microbiológica dessa bactéria durante a gestação e o início da antibioticoprofilaxia quando indicada, demonstrando a necessidade de adequar a prática de acompanhamento pré-natal às recomendações vigentes. $\bigcirc$ conhecimento sobre o perfil clínico-epidemiológico descrito nesta pesquisa pode contribuir para a elaboração de medidas preventivas locais.

Recomenda-se ainda a realização de novos estudos com um número amostral maior e com uma caracterização epidemiológica seguindo as recomendações vigentes, buscando correlacionar a colonização por EGB materna com prejuízos à saúde do feto.

\section{AGRADECIMENTOS}

À Faculdade de Ensino Superior da Amazônia Reunida e ao seu corpo docente, por proporcionarem condições necessárias para o alcance dos objetivos.

\section{APOIO FINANCEIRO}

Esta pesquisa foi realizada com recursos próprios.

\section{CONFLITO DE INTERESSES}

Os autores declaram não haver conflitos de interesse em relação à pesquisa.

\section{CONTRIBUIÇÃO DOS AUTORES}

Todos os autores contribuíram com a idealização do estudo, a análise e a interpretação dos dados e com a redação do manuscrito, aprovando a versão final publicada. Declaram ser responsáveis pelo conteúdo integral do artigo, garantindo sua precisão e integridade. 


\section{REFERÊNCIAS}

1 Melo SCCS, Santos NCS, Oliveira M, Scodro RBL, Cardoso RF, Pádua RAF, et al. Antimicrobial susceptibility of Streptococcus agalactiae isolated from pregnant women. Rev Inst Med Trop Sao Paulo. 2016 Nov;58:83.

2 Tortora GJ, Funke BR, Case CL. Microbiologia. 10. ed. Porto Alegre: Artmed; 2012. Capítulo 11. Procariotos: domínios Bacteria e Archaea; p. 299-328

3 Choi SY, Kim JW, Ko JW, Lee YS, Chang YP. Patterns of ischemic injury on brain images in neonatal group B Streptococcal meningitis. Korean J Pediatr. 2018 Aug;61 (8):245-52.

4 Centers for Disease Control and Prevention. Prevention of perinatal group B streptococcal disease: revised guidelines from CDC, 2010 [Internet]. Atlanta: Centers for Disease Control and Prevention; 2019 [cited 2019 Jan 12]. Available from: https://www.cdc.gov/mmwr/preview/ mmwrhtml/rr5910al.htm.

5 Sigaúque $B$, Kobayashi $M$, Vubil D, Nhacolo A, Chaúque $A$, Moaine $B$, et al. Invasive bacterial disease trends and characterization of group $B$ streptococcal isolates among young infants in southern Mozambique, 2001-2015. PLoS One. 2018 Jan; 13(1):e0191193.

6 Ministério da Saúde (BR). Secretaria de Atenção à Saúde. Departamento de Ações Programáticas Estratégicas. Gestação de alto risco: manual técnico. 5. ed. Brasília: Ministério da Saúde; 2010. (Série A. Normas e manuais técnicos).

7 Nkembe NM, Kamga HG, Baiye WA, Chafa AB, Njotang PN. Streptococcus agalactiae prevalence and antimicrobial susceptibility pattern in vaginal and anorectal swabs of pregnant women at a tertiary hospital in Cameroon. BMC Res Notes. 2018 Jul; $11(1): 480$.

8 Botelho ACN, Oliveira JG, Damasco AP, Santos KTB, Ferreira AFM, Rocha GT, et al. Streptococcus agalactiae carriage among pregnant women living in Rio de Janeiro, Brazil, over a period of eight years. PLoS One. 2018 May;13(5):e0196925.

9 Toyofuku M, Morozumi M, Hida M, Satoh Y, Sakata $H$, Shiro $H$, et al. Effects of intrapartum antibiotic prophylaxis on neonatal acquisition of group $B$ streptococci. J Pediatr. 2017 Nov;190:169-73.e1.

10 Ministério da Saúde (BR). Secretaria de Atenção à Saúde. Departamento de Atenção Básica. Atenção ao pré-natal de baixo risco. Brasília: Ministério da Saúde; 2012. (Série A. Normas e manuais técnicos. Cadernos de atenção básica; n. 32).
11 Costa ALRR, Araújo Jr E, Lima JWO, Costa FS. Maternal risk factors associated with the necessity of neonatal intensive care unit. Rev Bras Ginecol Obstet. 2014 Jan;36(1):29-34.

12 Hogan MC, Foreman KJ, Naghavi M, Ahn SY, Wang M, Makela SM, et al. Maternal mortality for 181 countries, 1980-2008: a systematic analysis of progress towards Millennium Development Goal 5. Lancet. 2010 May:375(9726):1609-23.

13 Le Doare K, O'Driscoll M, Turner K, Seedat F, Russell NJ, Seale AC, et al. Intrapartum antibiotic chemoprophylaxis policies for the prevention of group B streptococcal disease worldwide: systematic review. Clin Infect Dis. 2017 Nov;65 Suppl 2:S143-51.

14 Heath PT, Jardine LA. Neonatal infections: group B streptococcus. BMJ Clin Evid. 2014 Feb;2014:0323.

15 Rosini R, Margarit I. Biofilm formation by Streptococcus agalactiae: influence of environmental conditions and implicated virulence factors. Front Cell Infect Microbiol. 2015 Feb;5:6.

16 Zusman AS, Baltimore RS, Fonseca SNS. Prevalence of maternal group B streptococcal colonization and related risk factors in a Brazilian population. Braz J Infect Dis. 2006 Aug;10(4):242-6.

17 Instituto Brasileiro de Geografia e Estatística. Redenção [Internet]. Rio de Janeiro: IBGE; 2017 [citado 2019 set 3]. Disponível em: https://cidades. ibge.gov.br/brasil/pa/redencao/panorama.

18 Pará. Secretaria de Saúde Pública. Relatório anual de gestão do $12^{\circ}$ Centro Regional de Saúde/SESPA referente ao exercício 2016. Belém: Secretaria de Saúde Pública; 2017.

19 Brasil. Ministério da Saúde. Conselho Nacional de Saúde. Resolução $n^{\circ}$ 466, de 12 de dezembro de 2012. Estabelece diretrizes e normas regulamentadoras de pesquisas envolvendo seres humanos. Diário Oficial da União, Brasília (DF), 2013 jun 13; Seção 1:59.

20 Agência Nacional de Vigilância Sanitária (BR). Gram-positivos: Streptococcus spp [Internet]. Brasília: Agência Nacional de Vigilância Sanitária; 2008 [citado 2018 set 3]. Disponível em: http:// www.anvisa.gov.br/servicosaude/controle/rede_rm/ cursos/boas_praticas/modulo4/intr_stre.htm.

21 Clinical and Laboratory Standards Institute. Performance standards for antimicrobial susceptibility testing. CLSI supplement M100. 29th ed. Wayne (PA): Clinical and Laboratory Standards Institute; 2019. 
22 Magiorakos AP, Srinivasan A, Carey RB, Carmeli Y, Falagas ME, Giske CG, et al. Multidrug-resistant, extensively drug-resistant and pandrug-resistant bacteria: an international expert proposal for interim standard definitions for acquired resistance. Clin Microbiol Infect. 2012 Mar; 18(3):268-81.

23 Lima TM, Teles LMR, Oliveira AS, Campos FC, Barbosa RCC, Pinheiro AKB, et al. Corrimentos vaginais em gestantes: comparação da abordagem sindrômica com exames da prática clínica da enfermagem. Rev Esc Enferm USP. 2013 dez;47(6):1265-71.

24 Silva AK, Silva ADAT, Barros IM, Lima LR. Vulvovaginites durante a gestação e a importância do tratamento imediato - uma revisão de literatura. In: Anais do $12^{\circ}$ Encontro de Extensão, Docência e Iniciação Científica; 2016 dez; Quixadá, CE. Quixadá: Centro Universitário Católica de Quixadá; 2016.

25 Manning SD, Lewis MA, Springman AC, Lehotzky E, Whittam TS, Davies HD. Genotypic diversity and serotype distribution of group b streptococcus isolated from women before and after delivery. Clin Infect Dis. 2008 Jun;46(12): 1829-37.

26 Edwards MS, Rench MA, Palazzi DL, Baker CJ. Group B streptococcal colonization and serotype-specific immunity in healthy elderly persons. Clin Infect Dis. 2005 Feb;40(3):352-7.

27 Khan MA, Faiz A, Ashshi AM. Maternal colonization of group B streptococcus: prevalence, associated factors and antimicrobial resistance. Ann Saudi Med. 2015 Nov-Dec;35(6):423-7.

28 Batista RP, Ferreira CR. Streptococcus agalactiae septicemia in a patient with diabetes and hepatic cirrhosis. Autops Case Rep. 2015 Dec;5(4):35-43.

29 Wang YH, Su LH, Hou JN, Yang TH, Lin TY, Chu C, et al. Group B streptococcal disease in nonpregnant patients: emergence of highly resistant strains of serotype $\mathrm{lb}$ in Taiwan in 2006 to 2008. J Clin Microbiol. 2010 Jul;48(7):2571-4.

30 Pitts SI, Maruthur NM, Langley GE, Pondo T, Shutt KA, Hollick R, et al. Obesity, diabetes, and the risk of invasive group B streptococcal disease in nonpregnant adults in the United States. Open Forum Infect Dis. 2018 Jun;5(6):ofy030.

31 Silva P. Farmacologia. 8. ed. Rio de Janeiro: Guanabara Koogan; 2010. Seção 9, Antibióticos e quimioterápicos. Venenos animais, conceitos básicos da antibioticoterapia; p. 933-1005.

32 Linhares JJ, Cavalcante Neto PG, Vasconcelos JLM, Saraiva TV, Ribeiro AMF, Siqueira TM, et al. Prevalência de colonização por Streptococcus agalactiae em gestantes atendidas em maternidade do Ceará, no Brasil, correlacionando com os resultados perinatais. Rev Bras Ginecol Obstet. $2011 \mathrm{dez} ; 33(12): 395-400$.
33 Numanović F, Smajlović J, Gegić M, Delibegović Z, Bektaš S, Halilović $E$, et al. Presence and resistance of Streptococcus agalactiae in vaginal specimens of pregnant and adult non-pregnant women and association with other aerobic bacteria. Med Glas (Zenica). 2017 Feb;14(1):98-105.

34 Assefa S, Desta K, Lema T. Group B streptococci vaginal colonization and drug susceptibility pattern among pregnant women attending in selected public antenatal care centers in Addis Ababa, Ethiopia. BMC Pregnancy Childbirth. 2018 May; 18(1):135.

35 Kimura K, Suzuki S, Wachino J, Kurokawa H, Yamane K, Shibata N, et al. First molecular characterization of group B streptococci with reduced penicillin susceptibility. Antimicrob Agents Chemother. 2008 Aug;52(8):2890-7.

36 Seki T, Kimura K, Reid ME, Miyazaki A, Banno H, Jin $W$, et al. High isolation rate of MDR group $B$ streptococci with reduced penicillin susceptibility in Japan. J Antimicrob Chemother. 2015 Oct;70(10):2725-8.

37 Pereira JQ, Silva MT, Galvão TF. Use of antibiotics by adults: a population-based cross-sectional study. Sao Paulo Med J. 2018 Sep-Oct;136(5):407-13.

38 Arias B, Kovacec V, Vigliarolo L, Suárez M, Tersigni C, Müller L, et al. Fluoroquinolone-resistant Streptococcus agalactiae invasive isolates recovered in Argentina. Microb Drug Resist. 2019 Jun;25(5):739-43.

39 Bolukaoto JY, Monyama CM, Chukwu MO, Lekala SM, Nchabeleng M, Maloba MRB, et al. Antibiotic resistance of Streptococcus agalactiae isolated from pregnant women in Garankuwa, South Africa. BMC Res Notes. 2015 Aug;8:364.

40 Malheiro LF, Magano R, Ferreira A, Sarmento A, Santos L. Skin and soft tissue infections in the intensive care unit: a retrospective study in a tertiary care center. Rev Bras Ter Intensiva. 2017 Apr-Jun;29(2): 195-205.

41 Fiolo K, Zanardi CE, Salvadego M, Bertuzzo CS, Amaral E, Calil R, et al. Taxa de infecção e sorotipos de Streptococcus agalactiae em amostras de recém-nascidos infectados na cidade de Campinas (SP), Brasil. Rev Bras Ginecol Obstet. 2012 dez;34(12):544-9.

42 Prevention of group B streptococcal early-onset disease in newborns: ACOG committee opinion, number 797. Obstet Gynecol. 2020 Feb;135(2):e51-72.

43 Bianchi-Jassir F, Seale AC, Kohli-Lynch M, Lawn JE, Baker CJ, Bartlett L, et al. Preterm birth associated with group B streptococcus maternal colonization worldwide: systematic review and meta-analyses. Clin Infect Dis. 2017 Nov;65 Suppl 2:S133-42. 
44 Pintye J, Saltzman B, Wolf E, Crowell CS. Risk factors for late-onset group B streptococcal disease before and after implementation of universal screening and intrapartum antibiotic prophylaxis. J Pediatric Infect Dis Soc. 2016 Dec;5(4):431-8.
45 Prefeitura de São Paulo. Secretaria Municipal de Saúde. Coordenação de Epidemiologia e Informação. Alguns aspectos da evolução da mortalidade infantil na cidade de São Paulo. São Paulo: PMSP/SMS/CElnfo; 2009.

Recebido em / Received: 13/1 1/2019 Aceito em / Accepted: 26/2/2021 